\title{
The norms and singular numbers of polynomials of the classical Volterra operator in $L_{2}(0,1)$
}

\author{
by \\ Yuri Lyubich (Haifa) and Dashdondog Tsedenbayar (Ulaanbaatar)
}

\begin{abstract}
The spectral problem $\left(s^{2} I-\phi(V)^{*} \phi(V)\right) f=0$ for an arbitrary complex polynomial $\phi$ of the classical Volterra operator $V$ in $L_{2}(0,1)$ is considered. An equivalent boundary value problem for a differential equation of order $2 n, n=\operatorname{deg}(\phi)$, is constructed. In the case $\phi(z)=1+a z$ the singular numbers are explicitly described in terms of roots of a transcendental equation, their localization and asymptotic behavior is investigated, and an explicit formula for the $\|I+a V\|_{2}$ is given. For all $a \neq 0$ this norm turns out to be greater than 1 .
\end{abstract}

1. Introduction. For any compact linear operator $A$ in a Hilbert space the singular numbers $s_{k}(A)$ are the distances from $A$ to the set of all operators of rank less than or equal to $k-1, k \geq 1$. Their squares are the eigenvalues of the compact selfadjoint nonnegative operator $A^{*} A$ counted according to their multiplicities (see e.g. [1]). In particular, $s_{1}(A)=\|A\|$. The latter has been used by Halmos [2] to calculate the $L_{2}$-norm $\|\cdot\|_{2}$ of the classical Volterra operator

$$
(V f)(x)=\int_{0}^{x} f(t) d t, \quad 0 \leq x \leq 1 .
$$

Actually, the Halmos calculation yields

$$
s_{k}(V)=\frac{2}{(2 k-1) \pi}
$$

for all $k \geq 1$, in particular,

$$
\|V\|_{2}=2 / \pi
$$

The point is that the spectral problem

$$
\left(V^{*} V f\right)(x)=s^{2} f(x), \quad 0 \leq x \leq 1,
$$

2010 Mathematics Subject Classification: 47A10, 47A35, 47G10.

Key words and phrases: Volterra operator, singular numbers, boundary value problem. 
is equivalent to the boundary value problem

$$
f^{\prime \prime}(x)+\lambda f(x)=0, \quad f^{\prime}(0)=0, \quad f(1)=0,
$$

where $\lambda=1 / s^{2}$. This yields $f(x)=\cos \sqrt{\lambda} x$ (under the normalization $f(0)=1)$ and $\cos \sqrt{\lambda}=1$, which immediately implies (1.1).

A similar equivalence for the powers $V^{n}$ was established by Thorpe [4]. The corresponding boundary value problem is

$$
(-1)^{n} f^{(2 n)}=\lambda f, \quad f^{(l)}(0)=f^{(n+l)}(1)=0, \quad 0 \leq l \leq n-1 .
$$

In the present paper we generalize these results to the arbitrary complex polynomials

$$
\phi(V)=\sum_{i=0}^{n} a_{i} V^{i}, \quad a_{n} \neq 0, \quad n \geq 1 .
$$

Note that the operator $\phi(V)$ is not compact if $a_{0} \neq 0$, but in any case $\phi(V)^{*} \phi(V)=\left|a_{0}\right|^{2} I+K$ where $I$ is the identity operator and $K$ is a compact self-adjoint operator. This suggests defining the singular numbers of $\phi(V)$ as the nonnegative square roots of the eigenvalues of $\phi(V)^{*} \phi(V)$. In fact, they are positive, since the operator $\phi(V)$ is injective. Indeed, it is invertible if $a_{0} \neq 0$, otherwise, it is of the form $V^{l} \psi(V), l \geq 1$, with $\psi(V)$ invertible. Actually, the singleton $\left\{a_{0}\right\}$ is the spectrum of $\phi(V)$.

The singular numbers of $\phi(V)$ constitute a countable set $S_{\phi}$ converging to $\left|a_{0}\right|$. We have

$$
\sup S_{\phi}=\|\phi(V)\|_{2} \geq\left|a_{0}\right| .
$$

In Section 2 we consider the case $n=1$ and obtain an explicit formula for the singular numbers, in particular, for $\|I+a V\|_{2}$ ) in terms of roots of a transcendental equation that comes from a boundary value problem. We describe the localization of these roots in much detail. The general case $n \geq 1$ is considered in Section 3 where we construct a boundary value problem equivalent to the spectral problem in question. In Section 4 the problem of equality in (1.4) is discussed.

2. The case $n=1$. Let $\phi(V)=a_{0} I+a_{1} V, a_{1} \neq 0$. Since the case $a_{0}=0$ trivally reduces to that of [2], one can assume $a_{0} \neq 0$. Without loss of generality one can set $a_{0}=1$ and then denote $a_{1}$ by $a$, for short. Thus, we will consider $\phi(V)=I+a V$ with $a \neq 0$.

Our spectral problem

$$
\left(I+\bar{a} V^{*}\right)(I+a V) f=s^{2} f
$$

can be rewritten as

$$
\left(s^{2}-1\right) f-\bar{a} V^{*} f-a V f-|a|^{2} V^{*} V f=0 .
$$


We proceed from this integral equation to a differential equation by applying the operator $D=d / d x$ twice. Note that $D V=I$, while $D V^{*}=-I$ since

$$
\left(V^{*} f\right)(x)=\int_{x}^{1} f(t) d t, \quad 0 \leq x \leq 1 .
$$

Thus, 2.1) yields

$$
\left(s^{2}-1\right) f^{\prime}-(a-\bar{a}) f+|a|^{2} V f=0
$$

and then

$$
\left(s^{2}-1\right) f^{\prime \prime}-(a-\bar{a}) f^{\prime}+|a|^{2} f=0 .
$$

Now we insert $x=0$ and $x=1$ into 2.1 and 2.2 taking into account that

$$
(V f)_{x=0}=\left(V^{*} f\right)_{x=1}=0,
$$

and

$$
(V f)_{x=1}=\left(V^{*} f\right)_{x=0}=J_{1} \equiv \int_{0}^{1} f d t
$$

hence,

$$
\left(V^{*} V f\right)_{x=1}=0, \quad\left(V^{*} V f\right)_{x=0}=J_{2} \equiv \int_{0}^{1}(V f) d t .
$$

In this way we obtain four linear homogeneous equations for the six values: $f(0), f^{\prime}(0), f(1), f^{\prime}(1), J_{1}, J_{2}$. Eliminating $J_{1}$ and $J_{2}$ we get two boundary conditions for the differential equation (2.3). Let us emphasize that in [2] and [4] no integrals remain after substitution of $x=0$ and $x=1$ into the corresponding integral equation and its derivatives. In our case this is true only for one of the four equalities, namely, we get

$$
\left(s^{2}-1\right) f^{\prime}(0)-(a-\bar{a}) f(0)=0
$$

when putting $x=0$ in 2.2 . However,

$$
\left(s^{2}-1\right) f(1)-a J_{1}=0, \quad\left(s^{2}-1\right) f^{\prime}(1)-(a-\bar{a}) f(1)+|a|^{2} J_{1}=0
$$

for $x=1$ in 2.1 and 2.2), respectively. Eliminating $J_{1}$ we obtain

$$
\left(s^{2}-1\right) f^{\prime}(1)+\left(\bar{a} s^{2}-a\right) f(1)=0 .
$$

Lemma 2.1. For every $s$ the integral equation (2.1) is equivalent to the differential equation (2.3) with the boundary conditions (2.4) and 2.5).

Proof. We already know that each solution $f$ to the equation (2.1) satisfies $2.3-2.5$. In the converse direction we start with $f$ satisfying (2.3)(2.5) and set

$$
g=\left(\left(I+\bar{a} V^{*}\right)(I+a V)-s^{2} I\right) f .
$$


We have to show that $g=0$. To this end we note that $g^{\prime \prime}=0$ by $(2.3)$, hence $g(x)$ is a linear function of $x$. Furthermore,

$$
g^{\prime}(0)=\left(s^{2}-1\right) f^{\prime}(0)-(a-\bar{a}) f(0),
$$

hence $g^{\prime}(0)=0$ by (2.4). Thus, $g(x)$ is a constant, so

$$
\bar{a} g(1)=\bar{a} g(1)+g^{\prime}(1)=\left(s^{2}-1\right) f^{\prime}(1)-\left(\bar{a} s^{2}-a\right) f(1)
$$

by (2.5). Since $a \neq 0$, we get $g(1)=0$.

The singular numbers in question are just those $s$ for which the boundary value problem (2.3) $-(2.5)$ has a solution $f \neq 0$. It is easy to see that $s \neq 1$. Indeed, otherwise from (2.3) it follows that

$$
(a-\bar{a}) f^{\prime}-|a|^{2} f=0 .
$$

If $a$ is real then $f=0$ at once. If $a$ is not real then $f$ satisfies the linear differential equation (2.6) and, in addition, $f(0)=0$ by (2.4). Hence, $f=0$ again.

Now we are in a position to prove the following theorem.

TheOREM 2.2. Let $a=\alpha+i \beta$. The singular numbers of the operator $I+a V$ are given by the formula

$$
s=\sqrt{\frac{\alpha^{2}+\Delta}{\alpha^{2}+\beta^{2}}}
$$

where $\alpha$ and $\beta$ are the real and the imaginary parts of a and $\Delta$ runs over all real roots of the equation

$$
\sqrt{\Delta} \cot \frac{\sqrt{\Delta}\left(\alpha^{2}+\beta^{2}\right)}{\Delta-\beta^{2}}=\alpha .
$$

Though the value $\sqrt{\Delta}$ is determined only up to the factor \pm 1 , the righthand side of (2.8) is uniquely determined since the function $\cot (\cdot)$ is odd. Also note that if $\Delta$ is a root of the equation $(2.8)$ then

$$
\Delta \neq \beta^{2}, \quad \frac{\sqrt{\Delta}\left(\alpha^{2}+\beta^{2}\right)}{\Delta-\beta^{2}} \neq m \pi
$$

for all integers $m$, in particular, $\Delta \neq 0$. However, $\Delta=0$ becomes admssible by passing to the limit as $\Delta \rightarrow 0$. The limit equality is

$$
\frac{\beta^{2}}{\alpha^{2}+\beta^{2}}=-\alpha,
$$

or equivalently,

$$
\beta^{2}=-\frac{\alpha^{3}}{\alpha+1}
$$

where automatically $-1<\alpha<0$ and $\beta \neq 0$, so $a$ is not real. Under the relation 2.9 Theorem 2.2 extends by including $\Delta=0$ into 2.7), so we have 
the special singular number

$$
s^{0}=\frac{|\alpha|}{\sqrt{\alpha^{2}+\beta^{2}}}=\sqrt{1+\alpha}
$$

in this situation.

For $\Delta<0$ the equation 2.8 can be rewritten as

$$
\sqrt{|\Delta|} \operatorname{coth} \frac{\sqrt{|\Delta|}\left(\alpha^{2}+\beta^{2}\right)}{|\Delta|+\beta^{2}}=-\alpha .
$$

Proof of Theorem 2.2. In our current notation the equation $(2.3)$ is

$$
\left(s^{2}-1\right) f^{\prime \prime}-2 i \beta f^{\prime}+\left(\alpha^{2}+\beta^{2}\right) f=0,
$$

and the boundary conditions $(2.4)$ and $(2.5)$ are

$$
\left(s^{2}-1\right) f^{\prime}(0)-2 i \beta f(0)=0
$$

and

$$
\left(s^{2}-1\right) f^{\prime}(1)+\left(\left(s^{2}-1\right) \alpha-\left(s^{2}+1\right) i \beta\right) f(1)=0 .
$$

The characteristic equation for the differential equation 2.12 is

$$
\left(s^{2}-1\right) r^{2}-2 i \beta r+\left(\alpha^{2}+\beta^{2}\right)=0 .
$$

Its roots are

$$
r_{1}=i \frac{\beta+\sqrt{\Delta}}{s^{2}-1}, \quad r_{2}=i \frac{\beta-\sqrt{\Delta}}{s^{2}-1},
$$

where

$$
\Delta=\beta^{2}+\left(s^{2}-1\right)\left(\alpha^{2}+\beta^{2}\right) .
$$

The latter is equivalent to (2.7). We have to prove that 2.8) (including the equality $\Delta=0$ in the case $(2.9)$ ) is equivalent to the nontrivial solvability of the boundary value problem $2.12-(2.14)$. Let us start with $\Delta \neq 0$, i.e. $r_{1} \neq r_{2}$.

The general solution to 2.12$)$ is

$$
f(x)=c_{1} e^{r_{1} x}+c_{2} e^{r_{2} x},
$$

where $c_{1}$ and $c_{2}$ are arbitrary constants. Hence,

$$
f(0)=c_{1}+c_{2}, \quad f^{\prime}(0)=c_{1} r_{1}+c_{2} r_{2}
$$

and

$$
f(1)=c_{1} e^{r_{1}}+c_{2} e^{r_{2}}, \quad f^{\prime}(1)=c_{1} r_{1} e^{r_{1}}+c_{2} r_{2} e^{r_{2}} .
$$

By substitution into (2.13) and (2.14) we obtain a system of linear homogeneous equations for $c_{1}$ and $c_{2}$. This system has a nontrivial solution if and only if its determinant is equal to zero. This equality reduces to

$$
\left(\left(s^{2}-1\right) r_{1}-\alpha-i \beta\right) e^{r_{1}}=\left(\left(s^{2}-1\right) r_{2}-\alpha-i \beta\right) e^{r_{2}}
$$


by an elementary calculation taking (2.15) into account. Using (2.16) we obtain

$$
(\alpha-i \sqrt{\Delta}) e^{r_{1}}=(\alpha+i \sqrt{\Delta}) e^{r_{2}} .
$$

Note that $\alpha-i \sqrt{\Delta} \neq 0$, otherwise, $\alpha^{2}+\Delta=0$, which implies $s=0$ by (2.17). Therefore, one can rewrite (2.18) as

$$
e^{r_{1}-r_{2}}=\frac{\alpha+i \sqrt{\Delta}}{\alpha-i \sqrt{\Delta}}
$$

or equivalently,

$$
\frac{e^{r_{1}-r_{2}}+1}{e^{r_{1}-r_{2}}-1}=\frac{\alpha}{i \sqrt{\Delta}} .
$$

This is nothing but the equation (2.8) since

$$
r_{1}-r_{2}=\frac{2 i \sqrt{\Delta}}{s^{2}-1}=\frac{2 i \sqrt{\Delta}\left(\alpha^{2}+\beta^{2}\right)}{\Delta-\beta^{2}}
$$

by (2.16) and (2.17).

In the case $\Delta=0$ the only root of 2.15 is

$$
r=\frac{i \beta}{s^{2}-1}=-\frac{i\left(\alpha^{2}+\beta^{2}\right)}{\beta}
$$

since

$$
s^{2}-1=-\frac{\beta^{2}}{\alpha^{2}+\beta^{2}}
$$

by $(2.17)$. (Note that $\beta \neq 0$ since $s \neq 1$.) The general solution to 2.12 is now of the form

$$
f(x)=\left(c_{1}+c_{2} x\right) e^{r x} .
$$

Accordingly,

$$
f(0)=c_{1}, \quad f^{\prime}(0)=c_{1} r_{1}+c_{2} r_{2}
$$

and

$$
f(1)=c_{1} e^{r_{1}}+c_{2} e^{r_{2}}, \quad f^{\prime}(1)=c_{1} r_{1} e^{r_{1}}+c_{2} r_{2} e^{r_{2}} .
$$

It is easy to check that the determinant of the corresponding linear system for $c_{1}$ and $c_{2}$ vanishes if and only if the relation $(2.9)$ is valid. As we know, the latter is the limit form of 2.8 as $\Delta \rightarrow 0$.

Now we investigate the equation 2.8 with unknown $\Delta \neq 0$ (written as (2.11) for $\Delta<0$ ). We start with $\beta=0$. For the new unknown

$$
\xi=\alpha^{2} / \sqrt{|\Delta|}>0
$$

we have the equation

$$
\operatorname{coth} \xi=-\xi / \alpha
$$


if $\Delta<0$, but

$$
\cot \xi=\xi / \alpha
$$

if $\Delta>0$. By (2.7) and (2.19) the corresponding singular number is

$$
s=\sqrt{1+\frac{\varepsilon \alpha^{2}}{\xi^{2}}}
$$

where $\xi$ is a root of 2.20 or 2.21 and $\varepsilon=-1$ or 1 , respectively. By these equations one can rewrite 2.22 as

$$
s=1 / \cosh \xi \quad \text { or } \quad s=1 /|\cos \xi|,
$$

respectively.

The equation (2.20) has no positive roots if $\alpha>0$, but if $\alpha<0$ then 2.20 has exactly one positive root. Indeed, in the latter case the function $\operatorname{coth} \xi+\xi / \alpha$ on $(0, \infty)$ monotonically decreases from $+\infty$ to $-\infty$.

In contrast, the equation (2.21) has infinitely many positive roots for any $\alpha \neq 0$ : there is exactly one root $\xi_{k}$ of (2.21) in each interval $((k-1) \pi, k \pi)$, $k \geq 1$. Accordingly,

$$
s_{k}=\sqrt{1+\frac{\alpha^{2}}{\xi_{k}^{2}}}=\frac{1}{\left|\cos \xi_{k}\right|}, \quad k \geq 1 .
$$

Now let $\beta \neq 0$. Then we introduce the new unknown

$$
\xi=\sqrt{|\Delta|} /|\beta|>0,
$$

instead of $\Delta$, and the new real parameters

$$
\gamma=\frac{\alpha^{2}+\beta^{2}}{|\beta|}, \quad \delta=\frac{\alpha}{|\beta|},
$$

instead of $\alpha$ and $\beta$. In this setting we have

$$
\xi \operatorname{coth} \frac{\gamma \xi}{\xi^{2}+1}=-\delta
$$

if $\Delta<0$, but

$$
\xi \cot \frac{\gamma \xi}{\xi^{2}-1}=\delta
$$

if $\Delta>0$. The corresponding singular number is

$$
s=\sqrt{\frac{\delta^{2}+\varepsilon \xi^{2}}{\delta^{2}+1}}
$$

where $\xi$ is a root of 2.27) or (2.28) and $\varepsilon$ is defined as in 2.22).

Since $\gamma>0$, the equation 2.27) has no positive roots if $\delta \geq 0$. Let $\delta<0$, i.e. $\alpha<0$ by (2.26). Then all positive roots of (2.27) (if they exist) are less 
than $|\delta|$. With this restriction 2.27 is equivalent to $g(\xi)=0$ where

$$
g(\xi)=\frac{2 \gamma \xi}{\xi^{2}+1}-\log \frac{|\delta|+\xi}{|\delta|-\xi}
$$

The derivative $g^{\prime}(\xi)$ is

$$
\gamma\left(\xi^{2}-1\right)\left(\xi^{2}-\delta^{2}\right)-|\delta|\left(\xi^{2}+1\right)^{2}
$$

up to a positive factor. This biquadratic polynomial has at most two positive roots, so the same is true of $g^{\prime}(\xi)$ and hence of $g(x)$ because of Rolle's theorem and $g(0+)=0$. Thus, the equation (2.27) has at most two positive roots if $\delta<0$.

It remains to investigate the positive roots of the equation (2.28). With $\delta=0$ they are

$$
\xi_{k}^{0}=\frac{\gamma+\varepsilon_{k} \sqrt{\gamma^{2}+(2 k-1)^{2} \pi^{2}}}{(2 k-1) \pi}
$$

where $k$ runs over all integers and $\varepsilon_{k}=\operatorname{sign}(2 k-1)$. It is easy to see that $\xi_{k+1}^{0}<\xi_{k}^{0}$ for $k \neq 0$ but $\xi_{1}^{0}>\xi_{0}^{0}$. Moreover, $\xi_{1}^{0}>1$, while $\xi_{0}^{0}<1$. (It is useful to note that $\xi_{-(k-1)}^{0} \xi_{k}^{0}=1$.) Obviously, the roots $\xi_{k}^{0}$ and $\xi_{-k}^{0}$ tend to 1 as $k \rightarrow+\infty$.

Now let $\delta \neq 0$. Then with $\xi \neq \xi_{k}^{0}(k=0, \pm 1, \pm 2, \ldots), 2.28$ is equivalent to $h(\xi)=0$ where

$$
h(\xi)=h_{0}(\xi)-\arctan \left(\frac{\xi}{\delta}\right)-q(\xi) \pi, \quad h_{0}(\xi)=\frac{\gamma \xi}{\xi^{2}-1}
$$

and an integer coefficient $q(\xi)$ is determined by the inequality

$$
-\frac{\pi}{2}<h_{0}(\xi)-q(\xi) \pi<\frac{\pi}{2}
$$

The function $h_{0}(\xi)$ monotonically decreases from 0 to $-\infty$ on the interval $(0,1)$ and from $+\infty$ to 0 on the interval $(1, \infty)$. Since

$$
h_{0}\left(\xi_{k}^{0}\right)=\frac{(2 k-1) \pi}{2},
$$

we have $q(\xi)=k$ for $\xi_{k+1}^{0}<\xi<\xi_{k}^{0}, k \neq 0$, and $q(\xi)=0$ for $\xi<\xi_{0}^{0}$ or $\xi>\xi_{1}^{0}$. Thus,

$$
h(\xi)=h_{0}(\xi)-\arctan \left(\frac{\xi}{\delta}\right)-k \pi
$$

on the interval $J_{k}=\left(\xi_{k+1}^{0}, \xi_{k}^{0}\right), k \neq 0$, and

$$
h(\xi)=h_{0}(\xi)-\arctan \left(\frac{\xi}{\delta}\right)
$$

on the intervals $J_{0}=\left(0, \xi_{0}^{0}\right)$ and $J_{\infty}=\left(\xi_{1}^{0}, \infty\right)$. On each of these intervals 
the function $h(\xi)$ is continuous. Moreover, we have the one-sided limit values

$$
h\left(\xi_{k}^{0}-\right)=-\left(\frac{\pi}{2}+\arctan \left(\frac{\xi_{k}^{0}}{\delta}\right)\right)<0, \quad h\left(\xi_{k}^{0}+\right)=\frac{\pi}{2}-\arctan \left(\frac{\xi_{k}^{0}}{\delta}\right)>0
$$

for all $k$, and

$$
h(0+)=0, \quad h(+\infty)=-\frac{\pi}{2} \operatorname{sign} \delta .
$$

Therefore, the equation (2.28) has at least one root in each interval $J_{k}$, $k \neq 0, \infty$. For $k=\infty$ this is true if $\delta>0$. For $k=0$ it is true if $\delta<0$ and $|\delta| \gamma<1$. Indeed, in this case $h^{\prime}(0)>0$, so $h(\xi)>0$ for small $\xi>0$.

In general, the derivative $h^{\prime}(\xi)$ is

$$
\gamma\left(\xi^{2}+1\right)\left(\xi^{2}+\delta^{2}\right)+\delta\left(\xi^{2}-1\right)^{2}
$$

up to a negative factor. If $\delta>0$ then $h^{\prime}(\xi)<0$ for all $\xi>0$. In this case the equation $(2.28)$ has exactly one root $\xi_{k} \in J_{k}, k \neq 0$, including $J_{\infty}$, while $J_{0}$ does not contain roots at all.

If $\delta<0$ then $h^{\prime}(\xi)$ has at most two positive roots. These roots can lie either in a $J_{k}$, and then $(2.28)$ has no more than three roots there, or they belong to some different $J_{k}$ and $J_{l}$, and then the number of roots in each of them does not exceed 2. Any other interval $J_{m}$ contains exactly one root. The number of roots in $J_{0}$ and in $J_{\infty}$ does not exceed 2 because of (2.31).

Now we denote by $\xi_{k}$ a root of $h(\xi)$ in the interval $J_{k}$. The sequences $\left(\xi_{k}: k \geq 1\right)$ and $\left(\xi_{-k}: k \geq 0\right)$ monotonically tend to 1 from above and from below, respectively. By (2.29) with $\varepsilon=1$ the same is true for $\left(s_{k}: k \geq 1\right)$ and $\left(s_{-k}: k \geq 0\right)$. All these singular numbers are greater than $|\delta| / \sqrt{\delta^{2}+1}$. This lower bound is just the special singular number $s^{0}$ (see (2.10p) if $\Delta=0$ is a root of the equation (2.8), i.e. if (2.9) is valid. There are at most two singular numbers below this bound since they appear only if $\Delta<0$.

Theorem 2.3. Let $a=\alpha+i \beta \neq 0$. If $\beta=0$, i.e. $a=\alpha \in \mathbb{R} \backslash\{0\}$, then

$$
\|I+a V\|_{2}=\sqrt{1+\frac{\alpha^{2}}{\xi_{\min }^{2}}}=\frac{1}{\left|\cos \xi_{\min }\right|}
$$

where $\xi_{\min }$ is the smallest positive root of the equation (2.21).

If $\beta \neq 0$, then

$$
\|I+a V\|_{2}=\sqrt{\frac{\delta^{2}+\xi_{\max }^{2}}{\delta^{2}+1}}
$$

where $\xi_{\max }$ is the greatest root of the equation (2.28) with $\delta$ and $\gamma$ defined by 2.26).

Proof. Combine formula (1.4) with (2.24) in the case $\beta=0$ and with 2.29 in the case $\beta \neq 0, \varepsilon=1$. 
Corollary 2.4. If $\alpha=0$, i.e. $a=i \beta$, where $\beta \in \mathbb{R} \backslash\{0\}$, then

$$
\|I+a V\|_{2}=\frac{|\beta|}{\pi}+\sqrt{1+\frac{\beta^{2}}{\pi^{2}}} .
$$

Indeed, in this case we have 2.33 with $\delta=0$ and $\xi_{\max }=\xi_{1}^{0}$ coming from (2.30) with $\gamma=|\beta|$.

As $\beta \rightarrow \infty$, formula (2.34) leads to Halmos's formula (1.2). Similarly, (1.1) follows from (2.29) and (2.30).

COROLlary 2.5. The inequality

$$
\|I+a V\|_{2}>1
$$

holds for all complex $a \neq 0$.

Proof. This follows from 2.32 if $\beta=0$ and from 2.33 if $\beta \neq 0$ since $\xi_{\max }>1$ in either case.

Another way to get the inequality (2.35) (except for $a \leq 0$ ) is to recall that the operator $I+a V$ is not power bounded if $a$ is not real nonpositive (see [5]). With $a<0$ this operator becomes power bounded [5], but its norm remains greater than 1 by Corollary 2.5.

3. The general case. For an arbitrary polynomial $\phi$ of degree $n \geq 1$ we proceed from the integral equation

$$
\left(s^{2} I-\phi(V)^{*} \phi(V)\right) f=0
$$

to

$$
D^{2 n}\left(s^{2} I-\phi(V)^{*} \phi(V)\right) f=0,
$$

by differentiation of order $2 n$. In more detail, if $\phi(V)$ is of the form 1.3 then the equation (3.1) can be rewritten as

$$
\left(\left(s^{2}-\left|a_{0}\right|^{2}\right) I-\sum \bar{a}_{i} a_{k}\left(V^{*}\right)^{i} V^{k}\right) f=0
$$

with the summation over $0 \leq i, k \leq m, i+k \geq 1$. Accordingly, 3.2 takes the form

$$
\left(s^{2}-\left|a_{0}\right|^{2}\right) f^{(2 n)}-\sum_{j=1}^{2 n} c_{j} f^{(2 n-j)}=0
$$

where

$$
c_{j}=\sum_{i+k=j}(-1)^{i} \bar{a}_{i} a_{k} .
$$

Note that $c_{2 n}=(-1)^{n}\left|a_{n}\right|^{2} \neq 0$, so (3.3) cannot be an identity. This is a linear homogeneous differential equation with constant coefficients. The 
formal leading coefficient is $s^{2}-\left|a_{0}\right|^{2}$, so the order of the equation (3.3) is $2 n$ if $s \neq\left|a_{0}\right|$. The other cofficients do not depend on $s$.

Now we substitute $x=0$ and $x=1$ into the intermediate integrodifferential equations

$$
D^{i}\left(s^{2} I-\phi(V)^{*} \phi(V)\right) f=0, \quad 0 \leq i \leq 2 n-1,
$$

including (3.1). This yields $4 n$ linear homogeneous equations for $4 n$ boundary values

$$
f(0), f^{\prime}(0), \ldots, f^{(2 n-1)}(0), f(1), f^{\prime}(1), \ldots, f^{(2 n-1)}(1)
$$

and the integrals

$$
\left(\left(V^{*}\right)^{i} V^{k} f\right)(0), \quad\left(V^{k} f\right)(1), \quad 1 \leq i, k \leq n .
$$

The total number of these integrals is $n(n+2)$, but each one is a linear combination of $2 n$ power moments

$$
M_{l}=\int_{0}^{1} t^{l} f(t) d t, \quad 0 \leq l \leq 2 n-1 .
$$

Indeed, from the classical formula

$$
\left(V^{k} f\right)(x)=\frac{1}{(k-1) !} \int_{0}^{x}(x-t)^{k-1} f(t) d t
$$

and its version

$$
\left(\left(V^{*}\right)^{i} f\right)(x)=\frac{1}{(i-1) !} \int_{x}^{1}(t-x)^{i-1} f(t) d t
$$

it follows that

$$
\left(V^{k} f\right)(1)=\frac{1}{(k-1) !} \int_{0}^{1}(1-t)^{k-1} f(t) d t
$$

and

$$
\left(\left(V^{*}\right)^{i} V^{k} f\right)(0)=\frac{1}{(i-1) !(k-1) !} \int_{0}^{1} f(t) d t \int_{t}^{1}(s-t)^{k-1} s^{i-1} d s .
$$

All these integrals are linear combinations of $M_{l}, 0 \leq l \leq k-1$, and $M_{i+k-1}$. The orders of these moments are less than $2 n$.

Now let $F$ be the $4 n$-column consisting of the values $f^{(m)}(0)$ and $f^{(m)}(1)$, $0 \leq m \leq 2 n-1$, and let $M$ be the $2 n$-column consisting of the values $M_{l}$, $0 \leq l \leq 2 n-1$. Then we have an equation

$$
A F+B M=0
$$

where $A$ and $B$ are matrices of sizes $4 n \times 4 n$ and $4 n \times 2 n$, respectively.

To eliminate $M$ from (3.6) we take any $4 n$-row $w$ such that $w B=0$. Then we obtain a boundary condition $(w A) F=0$. In fact, only linearly 
independent systems of boundary conditions are interesting. Accordingly, we choose a basis $\left\{w_{j}: 1 \leq j \leq r\right\}$ in the space of solutions of the equation $w B=0$ and get

$$
\left(w_{j} A\right) F=0, \quad 1 \leq j \leq r .
$$

Note that $r \geq 2 n$ since the vector equation $w B=0$ can be rewritten as a system of $2 n$ linear homogeneous scalar equations with $4 n$ unknowns.

THEOREM 3.1. The integral equation (3.1) is equivalent to the differential equation (3.2) with the boundary conditions (3.7), where $r=2 n$ and $\left\{w_{j}\right\}$ is any linearly independent system of $2 n$ solutions of the equation $w B=0$.

Proof. We only need to prove that every solution $f$ of the boundary value problem under consideration satisfies (3.1). In other words, we have to prove that $g=0$ for

$$
g=\left(s^{2} I-\phi(V)^{*} \phi(V)\right) f .
$$

By (3.2) we have $D^{2 n} g=0$, therefore, $g$ is a polynomial of degree less than or equal to $2 n-1$. The column $G$ consisting of the values $g^{(m)}(0)$ and $g^{(m)}(1), 0 \leq m \leq 2 n-1$, satisfies the boundary conditions

$$
w_{j} G=0, \quad 1 \leq j \leq 2 n,
$$

since $G=A F+B M$ and $w_{j} A F=0$ by (3.7), while $w_{j} B=0$ by the choice of $w_{j}$.

The upper and the lower halves of the column $G$ are $G_{0}$ and $G_{1}$ whose entries are $g^{(m)}(0)$ and $g^{(m)}(1), 0 \leq m \leq 2 n-1$, respectively. The Taylor expansion at $x=0$ shows that $G_{1}=C G_{0}$ where $C$ is a $2 n \times 2 n$-matrix. The matrix $C$ is invertible by the Taylor expansion at $x=1$. The equations (3.8) can be rewritten as

$$
\left(u_{j}+v_{j} C\right) G_{0}=0, \quad 1 \leq j \leq 2 n,
$$

where $u_{j}$ and $v_{j}$ are the left and the right halves of the row $w_{j}$. From 3.9. it follows that $G_{0}=0$, and then $g=0$ since the rows $u_{j}+v_{j} C, 1 \leq j \leq 2 n$, are linearly independent. The latter is true since the rows $w_{j}, 1 \leq j \leq 2 n$, are linearly independent and the matrix $C$ is invertible.

By Theorem 3.1 the singular numbers of the operator $\phi(V)$ are just those $s$ for which the boundary linear functionals $F \mapsto\left(w_{j} A\right) F, 1 \leq j \leq 2 n$, on the space of solutions of the differential equation 3.2 are linearly dependent. Equivalently, the latter means that

$$
\operatorname{det}\left(w_{j} A F_{i}\right)=0
$$

where $\left\{f_{i}: 1 \leq i \leq 2 n\right\}$ is an arbitrary basis of that space (a "fundamental system" of solutions of (3.2)). Thus, the singular numbers are just the roots of the equation (3.10). The unknown $s$ is contained in the matrix $A$ and 
in the columns $F_{i}$ corresponding to the solutions $f_{i}, 1 \leq i \leq 2 n$. Actually, the matrix $A$ is lower triangular and all its diagonal entries are equal to $s^{2}-\left|a_{0}\right|^{2}$. The other entries of $A$ do not depend on $s$.

Theorem 3.1 also implies that for every singular number s the eigenspace of the operator $\phi(V)^{*} \phi(V)$ corresponding to the eigenvalue $s^{2}$ is of dimension $\leq 2 n$.

4. Appendix. An unsolved problem. According to Corollary 2.5 the only operator of $L_{2}$-norm 1 in the family $\{I+a V: a \in \mathbb{C}\}$ is $I$.

Problem. Describe the class $\mathcal{I}$ of functions $\phi(z)$ such that $\phi(0)=1$ and $\|\phi(V)\|_{2}=1$.

In this context $\phi(z)$ can be any function analytic in a neighborhood of $z=0, \phi(0)=1$. Since the spectrum of $\phi(V)$ is $\{1\}$ we have $\|\phi(V)\|_{2} \geq 1$ a priori.

The class $\mathcal{I}$ is not empty: the function $\mathbf{1}(z) \equiv 1$ belongs to $\mathcal{I}$. In total, $\mathcal{I}$ is a convex multiplicative semigroup. On the other hand, if $\phi \in \mathcal{I}$ and $\phi \neq \mathbf{1}$ then $\mathbf{1} / \phi \notin \mathcal{I}$, i.e. $\left\|\phi(V)^{-1}\right\|_{2}>1$. Indeed, otherwise, $\phi(V)$ is an isometry, hence $\phi(V)=I$ by the clasical Gelfand theorem.

For $\phi \in \mathcal{I}$ the operator $\phi(V)$ is power bounded. From Theorem 1.1 of [3] it follows that if $\phi \in \mathcal{I} \backslash\{\mathbf{1}\}$ then $\phi^{\prime}(0)$ is real negative. This necessary condition is not sufficient even for linear functions, as we already know. Moreover, we do not know any nontrivial polynomial $\phi \in \mathcal{I}$. We conjecture that there are no such polynomials. However, there are some functional examples.

PROpOSITION 4.1. If all roots of a polynomial $\phi$ are real negative and $\phi(0)=1$ then $\mathbf{1} / \phi \in \mathcal{I}$.

Proof. Halmos proved (see [2, Problem 150]) that $\left\|(I+V)^{-1}\right\|_{2}=1$. This means that the function $(1+z)^{-1}$ belongs to $\mathcal{I}$. The same proof shows that the function $(1+a z)^{-1}$ belongs to $\mathcal{I}$ for every $a>0$. The general case is settled by the decomposition of $\phi(z)$ into factors of the form $1+a z$ where $-a$ runs over the reciprocals of the roots of $\phi$.

REMARK 4.2. If $a$ is not real nonnegative then $\phi(z)=(1+a z)^{-1}$ does not belong to $\mathcal{I}$ since $\phi^{\prime}(0)=-a$. In other words, $\left\|(I+a V)^{-1}\right\|_{2}>1$ for all complex $a$, except for $a \geq 0$.

Halmos's proof cited above is based on the inequality $\operatorname{Re}(V f, f) \geq 0$ that means that the operator $-V$ is dissipative. Hence, if $a \leq 0$ then $\|\exp (a V)\|_{2} \leq 1$, so eventually $\|\exp (a V)\|_{2}=1$, thus $\exp (a z) \in \mathcal{I}$. The sufficient condition $a \leq 0$ is also necessary since $a=\phi^{\prime}(0)$.

Acknowledgments. The authors are grateful to Prof. Jaroslav Zemánek for useful discussions. 
The authors were supported by the European Community project "Operator theory methods for differential equations" (TODEQ).

\section{References}

[1] I. C. Gohberg and M. G. Kreŭn, Introduction to Theory of Linear Nonselfadjoint Operators, Transl. Math. Monogr. 18, Amer. Math. Soc., Providence, RI, 1969.

[2] P. R. Halmos, A Hilbert Space Problem Book, Van Nostrand, Princeton, 1967.

[3] Yu. Lyubich, The power boundedness and resolvent conditions for functions of the classical Volterra operator, Studia Math. 196 (2010), 41-63.

[4] B. Thorpe, The norm of powers of the indefinite integral operator in $(0,1)$, Bull. London Math. Soc. 30 (1998), 543-548.

[5] D. Tsedenbayar, On the power boundedness of certain Volterra operator pencils, Studia Math. 156 (2003), 59-66.

Yuri Lyubich

Technion

Haifa 32000, Israel

E-mail: lyubich@tx.technion.ac.il
Dashdondog Tsedenbayar

Department of Mathematics Mongolian University of Science and Technology

P.O. Box 46/520

Ulaanbaatar, Mongolia E-mail: tsdnbr@must.edu.mn 